\title{
Determination of Total Alkaloids in Different Parts of Actinidia arguta by Spectrophotography
}

\author{
Yang-yang Liü ${ }^{1, a}$, Chang-jiang Liu ${ }^{* b}$ \\ ${ }^{1}$ College of Food, Shenyang Agricultural University, Shenyang, 110866, China \\ * College of Food, Shenyang Agricultural University, Shenyang, 110866, China \\ ae-mail: juanjuanmao11@163.com be-mail: liucj597@sohu.com
}

Keywords: Actinidia arguta; Total Alkaloids; Spectrophotography

\begin{abstract}
Total Alkaloids are important active materials of Actinidia arguta. However, no study has been conducted to explore the content determination method of the Actinidia arguta total alkaloid. An effective method was established in order to determine the total alkaloids from different parts of Actinidia arguta. Using ultrasonic cleaner, the total alkaloids were extracted from the samples and tested at $415 \mathrm{~nm}$ with the spectroscopy by the means of acid dye method. The calibration curve of aconitine were linear $(\mathrm{r}=0.9997)$, and the recovery rate was prefect $(98.9 \%)$. The results of measurement were consistent in 3 hours. The content of alkaloids in roots was the highest, with $1.25 \mathrm{mg} / \mathrm{g}$, followed by leaves and fruits, the least were stems. The method is simple and effective, and the contents of total alkaloids were different in different parts of Actinidia arguta.
\end{abstract}

\section{Introduction}

Actinidia arguta belongs to Actinidiaceae; Actinidia is perennial and deciduous liana [1]. Actinidia arguta is famous for its cold resistance, the fruit is very small, like jujube, skin smooth and glabrous, direct edible [2]. The flavor is excellent, sweet, sour is moderate. It is an ideal green food and healthy food. The root, stem and fruit are all useful for us, because of their medical value and healthy function [3]. The Actinidia arguta is an important wild fruit resource and an ideal green healthy food. Actinidia arguta is a well-known traditional Chinese medicine which recorded in the Compendium of Materia Medica(Bencao Gangmu) by Li Shi-Zhen approximately 500 years ago [4].

Alkaloids are the active components of Chinese herbal medicine and medicinal plants, which have extensive biological activities [5]. Alkaloids have extensive bioactivities and pharmacological activities [6]. Total alkaloid content was determined form roots, stems, leaves and fruits of the Actinidia arguta. It can provide foundation and basis for comprehensive development and utilization of Actinidia arguta.

This experiment for the first time proposed the determination of the different parts of the alkaloids of Actinidia arguta. The content of different parts (roots, stems, leaves and fruits) of the total alkaloids of the Actinidia arguta have made a comprehensive analysis. The purpose is to find the alkaloid content of all parts, so it can provide a theoretical basis for the resources utilization of the new medicinal plant.

\section{Materials and Methods}

Primary materials and instruments. Roots, stems, leaves and fruits of the Actinidia arguta were purchased from the College of Food, Shenyang Agricultural University, China. UV- type 240 spectrophotometer (SHIMADZU Japan). Aconitine (American Sigma), other reagents are domestic analytical reagents.

The preparation of the sample solution. Using ultrasound, $5 \mathrm{~g}$ of dried powder of samples were extracted with $100 \mathrm{~mL}$ of chloroform 3 times at $40 \mathrm{C}$ for 1 hour. The chloroform extracts were evaporated to dryness by rotary evaporation at $60 \mathrm{C}$ under reduced pressure to produce a chloroform crude extract. The crude extract was dissolved by $10 \mathrm{~mL}$ chloroform. We put $1 \mathrm{~mL}$ liquid to a $10 \mathrm{~mL}$ 
volumetric flask and used the chloroform to constant volume. This was the sample solution. We used $\mathrm{NaOH}-\mathrm{KHC}_{8} \mathrm{H}_{4} \mathrm{O}_{4}$ for buffer solution. Fully shocked in separatory funnel with $0.5 \mathrm{~mL}$ of sample solution, $5.0 \mathrm{~mL}$ of buffer solution( $\mathrm{PH}=4), 2 \mathrm{~mL}$ of bromocresol green chromogenic agent and $9.5 \mathrm{~mL}$ of chloroform, and stewing 1 hour. Finally, the determination of absorbance values were used to the solution from the chloroform layer and joined the $\mathrm{Na}_{2} \mathrm{SO}_{4}$ for dehydration. Preparation of control check with distilled water in the same way.

The wavelength scanning. The standard solution, sample solution and control check solution were scanning from 350 to $500 \mathrm{~nm}$ for wavelength with chloroform as controls. The results showed that standard solution and sample solution all have maximum absorption (415 \pm 1$)$ and have the same absorption peak, almost no absorption peak of the control check at $415 \mathrm{~nm}$. So this experiment was chose $415 \mathrm{~nm}$ for measuring wavelength (Fig.1).

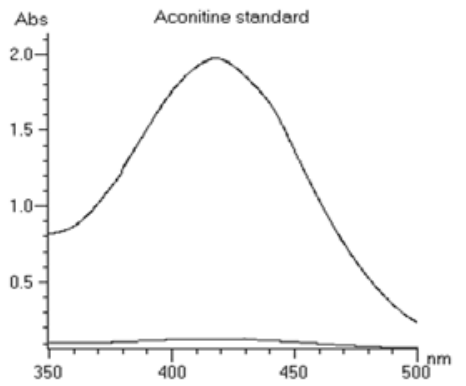

Fig. 1 The wavelength scanning figure

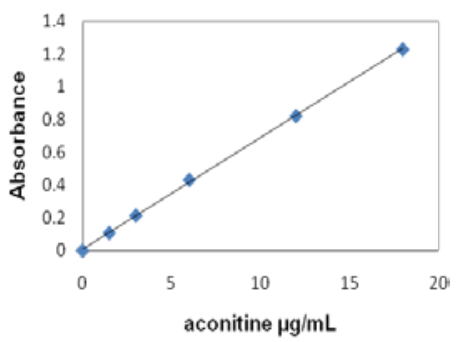

Fig.2 Aconitine standard curve

Stability test. The sample solution was determined the absorption values in $415 \mathrm{~nm}$ at room temperature. After testing, the standard solutions were placed in the freezer at 4C, 3 times/ hour, $\mathrm{RSD}=0.82 \%$.

Standard curve. 3mg of aconitine standard was dissolved to the scale with distilled water in $100 \mathrm{~mL}$ volumetric flask. Solution were extracted respectively $0,0.05,0.1,0.2,0.4,0.6 \mathrm{~mL}$ from the volumetric flask with distilled water to $1 \mathrm{~mL}$. Mixture to $10 \mathrm{~mL}$ with $5 \mathrm{~mL}$ of buffer solution $(\mathrm{PH}=4)$ $\mathrm{NaOH}-\mathrm{KHC}_{8} \mathrm{H}_{4} \mathrm{O}_{4}, 2 \mathrm{~mL}$ of bromocresol green chromogenic agent and chloroform were fully shaked well, and stewing 1 hour. Finally, the determination of absorbance values were used the solution from the chloroform layer and joined the $\mathrm{Na}_{2} \mathrm{SO}_{4}$ for dehydration. The regression equation $\mathrm{Y}=0.0682 \mathrm{x}+0.0077, \mathrm{r}=0.9997(\mathrm{n}=6)$, absorbance of aconitine show good linear relationship from $0-18.00 \mu \mathrm{g} / \mathrm{mL}$ (Fig.2)

Precision experiments. $0.2 \mathrm{~mL}$ of five copies of standard solution according to the preparation method of the sample solution for preparation of standard solution and blank solution measure the absorbance at $415 \mathrm{~nm}, \mathrm{RSD}=0.23 \%$, Show that precision is good.

Add sample recovery experiments. $5 \mathrm{~g}$ of dried powder of Actinidia arguta root added into the $1 \mathrm{~mL}$ of $300 \mu \mathrm{g} / \mathrm{mL}$ the aconitine standard according to the preparation method of the sample solution for preparation of standard solution and blank solution measure the absorbance at $415 \mathrm{~nm}$, The average recovery rate was $98.9 \%$, $\mathrm{RSD}=0.79 \%$.

\section{The total alkaloids of sample determination}

The total alkaloids of Actinidia arguta different parts (roots, stems, leaves and fruits) to the 2.2.1 method preparation of standard solution and blank solution measure the absorbance at $415 \mathrm{~nm}$. It can be seen that the larger difference of the content of alkaloids in different parts of the Actinidia arguta from table1. The content of alkaloids in roots was the highest, with $1.25 \mathrm{mg} / \mathrm{g}$. The alkaloid about $3 / 4$ of the roots, content difference between leaves and fruits were not significant $(\mathrm{P}>0.05)$. Stems, about $2 / 5$ of the roots, were the least alkaloid content. 
Table.1 The total alkaloid of Actinidia arguta different parts

\begin{tabular}{ccccc}
\hline Serial number & Parts & $\begin{array}{c}\text { Sample weight } \\
(\mathrm{g})\end{array}$ & Absorbance & $\begin{array}{c}\text { Total alkaloid C } \\
(\mathrm{mg} / \mathrm{g})\end{array}$ \\
\hline 1 & roots & 5.00 & 0.93 & $1.25^{\mathrm{a}}$ \\
2 & stems & 5.00 & 0.045 & $0.55^{\mathrm{c}}$ \\
3 & leaves & 5.00 & 0.072 & $0.95^{\mathrm{b}}$ \\
4 & fruits & 5.00 & 0.070 & $0.92^{\mathrm{b}}$ \\
\hline
\end{tabular}

The same column with different letters mean significant difference $(\mathrm{P}<0.05)$

\section{Conclusion}

Under the condition of certain $\mathrm{pH}$, alkaloids with hydrogen ion combined into salt. Acid dyes and the salt combined into non-ferrous complex, this complex can be extracted by organic solvent (chloroform). This experiment used aconitine as reference material [7-10]. Total alkaloid content was determined form roots, stems, leaves and fruits of the Actinidia arguta under the condition of $\mathrm{NaOH}-\mathrm{KHC}_{8} \mathrm{H}_{4} \mathrm{O}_{4}$ for buffer solution $\mathrm{pH}=4$ and bromocresol green for chromogenic agent[11]. This experiment also made a choice for different buffer solution and chromogenic agent. This study found that it was a strong stable property by the condition of $\mathrm{NaOH}-\mathrm{KHC}_{8} \mathrm{H}_{4} \mathrm{O}_{4}$ for buffer solution $\mathrm{pH}=4$ and bromocresol green for chromogenic agent in 3 hours. The $\mathrm{pH}$ value has a great influence on chromogenic agent. Compared with liquid chromatography (HPLC), experimental results show that this method is rapid, reliable, high sensitivity and using less dosage of the sample.

From the perspective of the determination results of alkaloid, roots, stems, leaves and fruits of the Actinidia arguta all have alkaloid. It is larger difference of the content of alkaloids in different parts of the Actinidia arguta. The content of alkaloids in roots was the highest, with $1.25 \mathrm{mg} / \mathrm{g}$, followed by leaves and fruits and the least was stems. But the content of leaves and fruit is essentially the same. This study can provide the basis for the medicinal value of the fruit. This builds lays a foundation for the comprehensive development and utilization of the Actinidia arguta.

\section{Acknowledgement}

In this paper, the research was sponsored by The ministry of agriculture of national public welfare industry project (Project No. 200903013).

\section{References}

[1] Li Xuan. Antioxidant Activity and Immunity Activity of the Polysaccharides from Actinidia arguta [D]. Shenyang Agricultural University, 2013 17-22.

[2] Yongri Jin, The Research of Chemical Composition of Actinidia arguta Roots [J]. Pharmaceutical Journal of China, 1998 33(7) 402.

[3] Deqiu Zhu, Zhongshen Liu. The Research of Actinidia arguta Anti-aging Effect [J]. Journal of Hunan Medical College, 1996 2(2) 54-57.

[4] Li shizhen original. The illustration Compendium of Materia Medica [C]. 2007.

[5] Huzhan Zheng. Application and Research of Modern Chinese Medicine (The third volume) [C]. 2275-2729.

[6] Wink M, Roberts M F. Compartmentation of Alkaloid Synthesis, transport and storage. In: 
Robers M F, Wink M. Alkaloids: Biochemistry, Ecology and Medicinal Applications [M]. New York and London: Plenum Press, 1998 239-262.

[7] Zhao Boguang. Nematicidal Activity of Quinolizidine Alkaloids and the Functional Group Pairs in Molecular Structure[J]. Journal of Chemical Ecology, 199925 (10) 2205-2214.

[8] Gonzalez-Coloma.A, et.al. Stucture Diversity and Defensive Properties of Norditerpenoid Alkaloids [J]. Journal of Chemical Ecology, 2004 30(7) 1393-1408.

[9] Jianming Huang, Jixian Guo, Mingming Sun. The Study of the Determination Method of Alkaloids Contained in Kusnezoff Monkshood [J]. Traditional Chinese Medicine, 2002 25(12) 878

[10] Reina M, Gonzalez Coloma A, Gutierrez C, et.al. Pyrrolizidine Alkaloids from Heliotropisms' Megalanthum [J]. The Journal of Natural Products, 1998 61(11) 1418- 1420.

[11] Jianhong Zeng, Zhengsong Peng, Jingyuan Song. The Dynamic Change of Pinellia Total Alkaloid Content [J].Traditional Chinese Medicine, 2004 27(7) 471. 